\title{
Inferior vena cava parameters predict re-admission in ischaemic heart failure
}

\author{
Federico Carbone* ${ }^{*}$, Marta Bovio*, Gian Marco Rosa ${ }^{\ddagger}$, Fabio Ferrando* ${ }^{*}$ Alberto Scarrone*, \\ Giovanni Murialdo* ${ }^{*}$, Alessandra Quercioli ${ }^{\dagger}$, Nicolas Vuilleumier ${ }^{\S}$, François Mach ${ }^{\dagger}$, Francesca Viazzi ${ }^{\ddagger}$ and Fabrizio \\ Montecucco*,
}

*Department of Internal Medicine, University of Genoa School of Medicine, IRCCS Azienda Ospedaliera Universitaria San Martino-IST Istituto Nazionale per la Ricerca sul Cancro, Genoa, Italy, ${ }^{\dagger}$ Division of Cardiology, Foundation for Medical Researches, Department of Medical Specialties, University of Geneva, Geneva, Switzerland, "'Departments of CardioNephrology University of Genoa, School of Medicine, IRCCS Azienda Ospedaliera Universitaria San Martino-IST Istituto Nazionale per la Ricerca sul Cancro, Genoa, Italy, ${ }^{\S}$ Division of Laboratory Medicine, Department of Genetics and Laboratory Medicine, Geneva University Hospitals, Geneva, Switzerland

\begin{abstract}
Background The clinical history of heart failure (HF) is usually characterized by frequent hospitalizations for decompensation. Therefore, several markers of subclinical hemodynamic congestion are under investigation for predicting early rehospitalization. In this field, the potential of ultrasound inferior vena cava (IVC) assessment has been recently investigated in HF but not yet assessed in the different aetiological categories.
\end{abstract}

Material and methods Forty-eight patients admitted for decompensated HF ( $n=25$ with ischaemic heart disease [IHD] and $n=23$ non-IHD) underwent biochemical examination (including NT-proBNP), echocardiography and IVC assessment by hand-carried ultrasound (HCU). During 60-day follow-up after discharge, the re-hospitalization rate for HF was recorded to investigate the predictive power of NT-proBNP and IVC assessment among the two study groups.

Results IHD and non-IHD patients with HF were similar except for gender distribution. During follow-up, $16 \cdot 7 \%$ of patients were rehospitalized for decompensated HF, with higher prevalence in IHD group (28\% vs. $4.3 \%$ $P=0.031)$. IVC assessment at discharge significantly predicted re-admission in the overall population and in IHD group, whereas NT-proBNP failed to predict rehospitalization in IHD group. In adjusted hazard ratio, only IVC min and the changes of IVC from admission significantly predicted re-admission. ROC analysis confirmed the change in IVC min as the best predictor of rehospitalization in patients with IHD.

Conclusion This pilot study showed a higher early re-admission rate in patients with HF due to IHD. In addition, the change in IVC min diameter from admission to discharge was the best predictor of re-admission in patients with IHD.

Keywords Chronic heart failure, echography, inferior vena cava, ischaemic heart disease.

Eur J Clin Invest 2014; 44 (4): 341-349

\section{Introduction}

Acute decompensated heart failure (ADHF) results from the rapid worsening of chronic heart failure ( $\mathrm{CHF})$ [1] and is more often due to increased congestion and volume overload rather than a pure low cardiac output [2-4]. In general, ADHF requires the hospitalization independently on its pathophysiology and aetiology. In fact, a cluster of heterogeneous diseases [including ischaemic heart disease (IHD), myocarditis and other non-IHD causes] [5] has been described to induce a similar final CHF. However, heart failure (HF) secondary to
IHD was recently associated with a worse long-term outcome in terms of both hospitalization [6] and mortality [7]. Considering that clinical re-admissions after discharge is particularly frequent, the identification of predictive parameters in different HF conditions might be useful to the management of this disease [8]. As persistent hemodynamic congestion has been indicated as the most important determinant to readmission [9], some promising biomarkers and imaging technologies, able to subclinically detect it [10], are under investigation to be validated in the clinical practice. For instance, elevated right atrial pressure (RAP) [11], left ventricular 
ejection fraction (LVEF) [12], left ventricular end-diastolic volume index [13], together with serum levels of natriuretic peptide assay [14], have been indicated as useful HF prognostic parameters alone or in combination. Recently, the RAP estimation by hand-carried ultrasound (HCU) assessment of inferior vena cava (IVC) size and collapsibility [15] has been shown to correlate with brain natriuretic peptide (BNP) in a cohort of patients with abnormal LV filling pressures [16]. Furthermore, recent advances in ultrasound device development have broadened and simplified this approach. In particular, with both HCU [17-19] and new hand-held devices [20], ultrasound examination was shown feasible by noncardiologist, also concerning the assessment of IVC size and collapsibility [21-25]. Considering these findings, the aim of this study was to assess the predictive value of IVC measurement (including diameters and collapsibility performed both at admission and discharge) on 60-day rehospitalization in different categories of patients with $\mathrm{CHF}$, such as $\mathrm{HF}$ due to chronic IHD or to non-IHD.

\section{Methods}

The Medical Ethics Committee of IRCCS Azienda Ospedaliera Universitaria San Martino-IST Istituto Nazionale per la Ricerca sul Cancro approved this study, and participants provided written informed consent before enrolment. The study was conducted in compliance with the Declaration of Helsinki.

\section{Patient population}

All patients consecutively admitted to the Department of Internal Medicine of IRCCS Azienda Ospedaliera Universitaria San Martino-IST Istituto Nazionale per la Ricerca sul Cancro in Genoa, Italy, with a primary diagnosis of ADHF during the study period (from November 2011 to January 2013) were prospectively screened. In according to the guidelines of European Society of Cardiology, the diagnosis of ADHF was performed by medical history and physical examination, in addition to NT-proBNP assay [5]. CHF was classified as due to IHD or non-IHD, according to the definition of the International Classification of Diseases-10 [26]. In particular, as previously described [27], the IHD group included only patients with a history of previous myocardial infarction or revascularization. Exclusion criteria were the admission for rapid deterioration induced by acute coronary syndrome and its related mechanical complications, rapid arrhythmia or severe bradycardia, acute pulmonary embolism, hypertensive crisis, aortic dissection and surgery/perioperative problems [5]. Other exclusion criteria were concomitant myocarditis, pregnancy, amyloidosis, constrictive pericarditis, pericardial effusion, high output states (severe anaemia, sepsis, thyrotoxicosis, Paget's disease), renal replacement therapy, chronic hepatitis, acute and chronic infections, autoimmune rheumatic disease and cancer. Finally, forty-eight patients ( $n=25$ IHD and $n=23$ non-IHD, respectively) were included in the study. Biochemical analysis and HCU assessment of congestion were performed on admission and at discharge. Patients were prospectively followed up for 60 days after discharge by a weekly telephone interview. At the time of re-admission, all the clinical files of patients were analysed to establish whether the cause of rehospitalization was related to ADHF.

\section{Study power calculation}

Although this is a 'pilot' study, the sample size was computed based on an expected prevalence of re-admission for ADHF in patients with HF due to IHD or non-IHD. A minimum of 1.6-fold increased rehospitalization risk was observed in patients with IHD admitted for ADHF and developed combining the results of a previous published perspective study [28]. This study reported that an ischaemic aetiology of CHF was independently associated with 1.75 -fold increased risk of re-admission within 30 days and 1.60 within 90 days [28]. According to our power calculation for log-rank test, the minimal sample size request to detect a 1.6-fold increase in the risk of re-admission with a power of $95 \%$ and with a two-sided alpha error of $5 \%$ was of 24 patients.

\section{Clinical and biochemical assessments}

Physical examination, cardiac medications and laboratory parameters were recorded at admission and at discharge after being rendered anonymous. New York Heart association (NYHA) class was assessed by the same investigator observing each patient at rest and walking [5]. Plasma NTproBNP values were determined at admission and discharge by enzyme immunoassay (Siemens Healthcare Diagnostic Inc., Erlangen, Germany) in a single hospital laboratory. The lower range value for NT-proBNP was $5 \mathrm{ng} / \mathrm{L}$. Intra- and interassays coefficient of variations were $6 \%$ and $7.7 \%$, respectively.

\section{Echographic evaluation}

Within $12 \mathrm{~h}$ from admission and the day of the discharge, all patients underwent a bedside HCU evaluation to measure IVC parameters (maximal and minimal diameters of IVC throughout the respiratory cycle (IVCmax and IVCmin, respectively)). All the echographic examinations were carried out by the same investigator (who was blinded to other clinical and laboratory evaluations) using UF-750XT device (Fukuda Denshi USA, Inc., Redmond, WA, USA). In turn, the treating physicians were blinded to the results of ultrasound assessment. Considering that previous researches have demonstrated a $90 \%$ success rate for physicians with poor training in HCU assessment of IVC [17-19], the echography operator in this study 
was a resident in internal medicine with a formal training in ultrasonography. As previously described [29], IVC diameter was measured from a subcostal approach $2 \mathrm{~cm}$ before to merge in the right atrium. In addition, all measurements were taken during diastole, when the blood flow from IVC to right atrium is at minimum. The rate of changes in IVC diameters during respiration, called IVC collapsibility, was recorded and calculated as [(IVCmax-IVCmin)/IVCmax] $\times 100$. The changes of IVC diameters during the hospitalization were expressed as percentage (\%) rate of [(IVC admission-IVC discharge)/IVC admission]. A transthoracic echocardiography assessing the cardiac structure and function was also performed, once patients were stabilized, as recently suggested [30]. In particular, NYHA class II was established as cut-off point defining clinical stabilization. Echocardiography was performed by one experienced operator (a cardiologist blinded to the clinical and biochemical results). As recommended by American Society of Echocardiography [31], analysis of left ventricle (LV) included linear dimension and volumes (systolic and diastolic), wall thickness and mass (both crude and indexed for body surface area) as well as a functional evaluation of LVEF by the Simpson method. Also, diastolic LV function was assessed by measuring the mitral inflow velocities (E/E' ratio and deceleration time of $\mathrm{E}$ wave). Furthermore, right ventricular structure and function were investigated by right ventricle diameter measurement, the estimations of systolic pulmonary artery pressure (PAPS) and tricuspid annular plane systolic excursion (TAPSE) [31].

\section{Statistical analysis}

Statistical analyses were performed using SPSS 20.0 statistical package (SPSS Inc., Chicago, IL, USA). Categorical data are presented as percentage, whereas quantitative continuous variables were expressed as medians (interquartile range). For statistical analysis, BNP values were log-transformed to reduce the typical skewness recognized in our cohort by Shapiro-Wilk test as already reported in the literature [32]. Therefore, statistical differences between the two groups (IHD and non-IHD) were assessed with the use of MannWhitney test and chi-square analysis. Kaplan-Meier curves with log-rank statistic were used to illustrate the outcome, whereas the predictors of re-admission were identified by univariate and multivariate Cox proportional hazard regression analyses. The prognostic ability of noninvasive assessment of hemodynamic congestion was further assessed by the receiver operator curves (ROC). The area under the curve (AUC) was given with 95\% confidence interval (CI), and cutoff points were calculated maximizing the sensitivity. The AUCs of two curves were compared with the DeLong's method [33]. A 2-sided P-value $<0.05$ was considered statistically significant.

\section{Results}

\section{Patient characteristics at admission and discharge are comparable in HF patients due to IHD or non-IHD}

Baseline clinical and laboratory characteristics of patients with IHD and non-IHD HF are presented in Supplementary Table S1. As previously reported [34], the male gender was prevalent in the IHD group as compared to non-IHD (72\% vs. $28 \%$; $P=0.009)$. At admission, no other significant differences in term of age, comorbidities or hospitalization time (Table S1) were shown between the two HF groups. At discharge, serum uric acid concentration was significantly increased in patients with non-IHD as compared with IHD (Table 1). No other biochemical [including haemoglobin $(\mathrm{Hb})$, neutrophil/lymphocyte $(\mathrm{N} / \mathrm{L})$ ratio and creatinine] or clinical (NYHA class distribution, systemic blood pressure, weight loss from admission and hospitalization time) parameter was significantly different between both groups (Table 1). Importantly, at discharge, medications administered to patients with IHD were similar to those of non-IHD group (Table 1). The echocardiographic examination did not show any difference between the two study groups (Table S2). Considering biomarkers of HF, NT-pro-BNP levels at discharge and NT-pro-BNP changes from admission were comparable in patients with IHD and non-IHD (Table 1). When comparing admission and discharge values of IVC size and collapsibility, significant reduction in IVC max and IVC min (Fig. 1a, b), as well as increased IVC collapsibility was shown at discharge (Fig. 1c) in both IHD and non-IHD groups. However, no difference in IVC size and collapsibility values was shown between the two study groups at each time point (Fig. 1a-d).

IVC parameters predict rehospitalization in overall HF cohort and in patients with IHD, but not in non-IHD During the 60-day follow-up, 8 patients (16.7\%) were rehospitalized for ADHF on the total cohort of patients $(n=48)$. The Kaplan-Meier analysis showed that patients with non-IHD were less re-admitted for ADHF as compared to IHD group (re-admission rate in non-IHD vs. IHD: $4.3 \%$ vs. $28 \%$; $P=0.031$ ) (Fig. 2). Cox proportional hazard model was performed to define whether biochemical or echography-assessed hemodynamic congestion at discharge predicted re-admission for ADHF at 60 days from discharge. At univariate analysis, higher plasma levels of $\log (\mathrm{NT}$-proBNP) at discharge were statistically significant predictors of re-admission [hazard ratio (HR) 1.77, 95\% CI: 1.05-2.98; Wald $\chi^{2}=4.67 ; P=0.03$ ] in the overall population but not in single IHD and non-IHD groups (Table S3). IVC max and min diameters as well as IVC collapsibility at discharge significantly predicted re-admission in the overall population and in IHD, but not in non-IHD group 
Table 1 Clinical, laboratory characteristics and medications of study population at discharge

\begin{tabular}{|c|c|c|c|}
\hline & Patients with non-IHD & Patients with IHD & $\boldsymbol{P}$-value \\
\hline \multicolumn{4}{|l|}{ Laboratory characteristics } \\
\hline $\mathrm{Hb}, \mathrm{g} / \mathrm{dL}$ (IQR) & $12 \cdot 1(11 \cdot 3-13 \cdot 4)$ & $11.5(10.7-12.9)$ & 0.224 \\
\hline N/L ratio, no. (IQR) & $3(2-4)$ & $3(2-6)$ & 0.659 \\
\hline Creatinine, mg/dL (IOR) & $1.2(0.9-1.5)$ & $1.3(1.0-1.6)$ & 0.527 \\
\hline Uric acid, mg/dL (IQR) & $7.58(6.30-8.24)$ & $5 \cdot 87(5 \cdot 19-7.22)$ & 0.015 \\
\hline NT-proBNP, ng/L (IOR) & $2360(1080-4542)$ & 3903 (1138-12386) & 0.291 \\
\hline Changes in NT-proBNP\% (IQR) & $3.34(-0.07-10.77)$ & $4.47(1.68-13.01)$ & 0.336 \\
\hline \multicolumn{4}{|l|}{ CHF clinical features } \\
\hline NYHA class II, no. (\%) & $19(86 \cdot 4)$ & $18(72.0)$ & 0.297 \\
\hline NYHA class III, no. (\%) & $3(13.0)$ & $3(12.0)$ & 0.913 \\
\hline NYHA class IV, no. (\%) & $0(0.0)$ & $0(0.0)$ & 1.000 \\
\hline Systolic BP, mmHg (IQR) & $125(115-130)$ & $130(120-140)$ & 0.209 \\
\hline Diastolic BP, mmHg (IQR) & $70(62-80)$ & $70(65-80)$ & $0 \cdot 737$ \\
\hline Weight reduction from admission,\% (IQR) & $4.7(2.5-6 \cdot 6)$ & $2.5(0.0-5.4)$ & 0.070 \\
\hline Hospitalization time, days (IQR) & $10(6-14)$ & $8(7-11)$ & $0 \cdot 216$ \\
\hline \multicolumn{4}{|l|}{ Medications } \\
\hline RAAS inhibitors, no. (\%) & $17(73.9)$ & $17(68.0)$ & 0.756 \\
\hline Beta-blockers, no. (\%) & $15(65 \cdot 2)$ & $18(72 \cdot 0)$ & $0 \cdot 757$ \\
\hline Aldosterone antagonist, no. (\%) & $12(52 \cdot 2)$ & $11(44 \cdot 0)$ & 0.773 \\
\hline Loop diuretics, no. (\%) & $19(82 \cdot 6)$ & $20(80 \cdot 0)$ & 1.000 \\
\hline Calcium channel blockers, no. (\%) & $3(13 \cdot 0)$ & $4(16 \cdot 0)$ & 1.000 \\
\hline Antiplatelets, no. (\%) & $10(43.5)$ & $17(68.0)$ & $0 \cdot 145$ \\
\hline
\end{tabular}

The bold values identify the statistically significant results $(P<0.05)$.

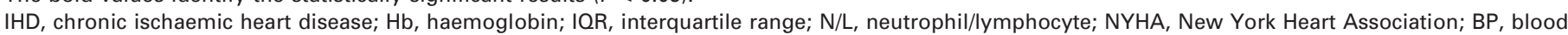
pressure; RAAS, renin-angiotensin-aldosterone system.

(Table S3). Because of low numbers of events $(n=8)$, only two covariates were computed in multivariate analysis. Thus, we selected as covariates those that statistically differed between the two groups in the descriptive analysis: gender and levels of uric acid at discharge. This was in agreement with previously reported, especially for high levels of uric acid, higher in patients with IHD [35] and also related to a worse outcome of HF [36]. In the adjusted analysis, $\log (\mathrm{NT}$-proBNP) and IVC diameters, collapsibility and change from admission at discharge maintained the statistical significance to predict re-admission in the overall population (Table 2). In the IHD group, only IVC min and IVC change from admission significantly predicted re-admission for ADHF (Table 2). In particular, when expressed as percentage (\%) rate, the change from admission in IVC min diameters was shown as the best predictor of re-admission at 60 days from discharge in both overall cohort and IHD group (Table 2). ROC analysis showed the high statistical sensitivity and specificity of NT-proBNP in predicting re-admission for ADHF in the overall CHF cohort, but not in IHD group (Table S4). Likewise, also HCU discharge assessment of IVC max, IVC min, IVC collapsibility and changes in IVC from admission had a good predictive values in both overall HF cohort and IHD group (Table S4). Comparison of ROC of NT-proBNP, IVC max and change in IVC min in patients with IHD confirmed the best sensitivity and specificity values for change in IVC min (Fig. 3). In addition, the ROC analysis provided the cut-off values for a better prediction of rehospitalization (Table S4). The cut-off values of $1.9 \mathrm{~cm}$ for 
(a)
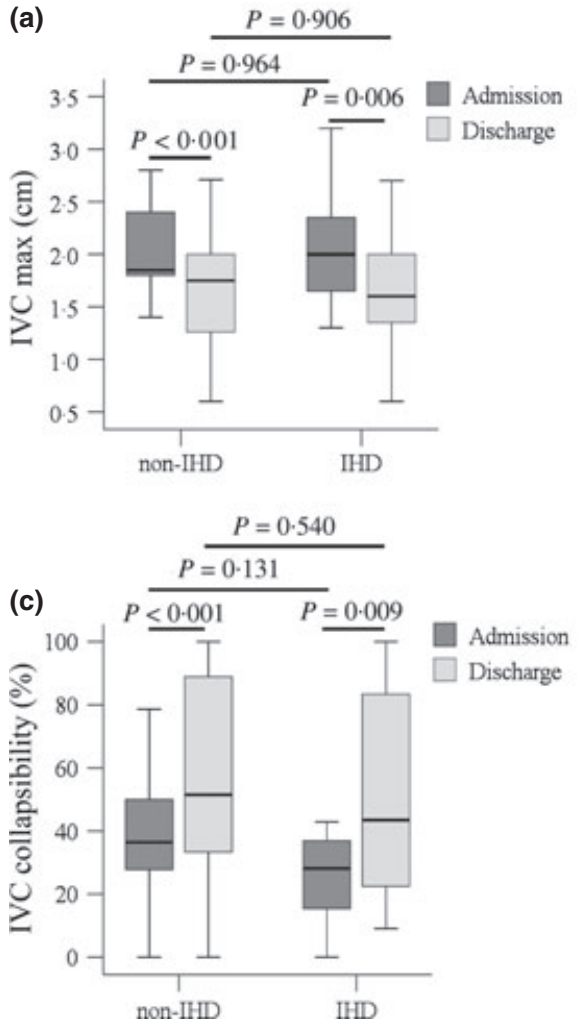

(b)
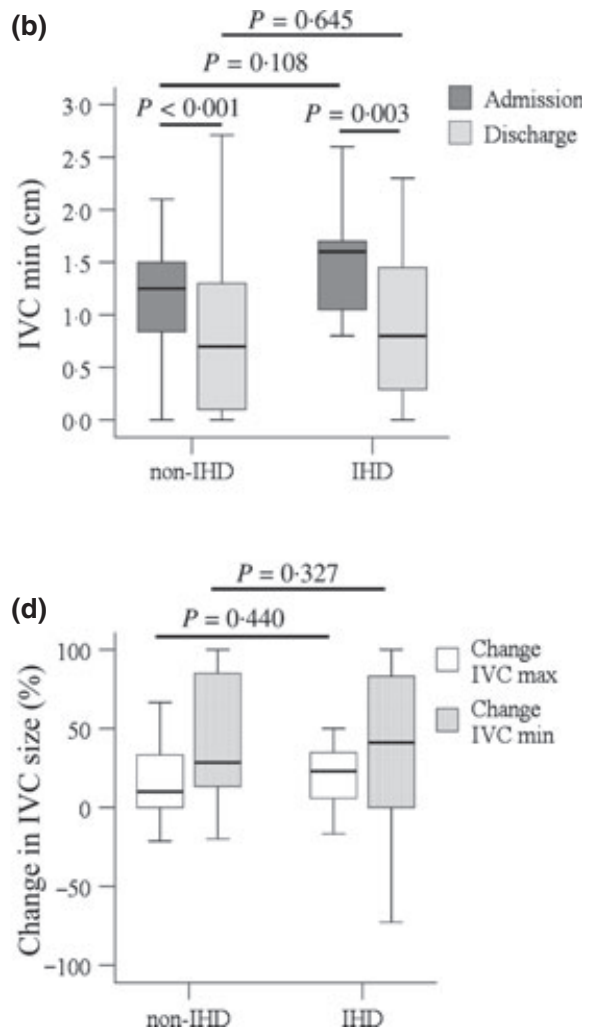

Figure 1 IVC size and collapsibility at admission and discharge in patients with IHD and non-IHD. During hospitalization, there was a significant reduction in inferior vena cava (IVC) size (including IVC maximum [a] and minimum [b] diameters) and a significant increase in IVC collapsibility (c) in both patients with IHD and non-IHD. Changes from admission to discharge in IVC max and IVC min were not significantly different between groups (d). Data are shown as median (25th-75th interquartile range).

IVC max, $50 \%$ for IVC collapsibility in the overall population were in accordance with previous reports [15, 29] (Table S4). Likewise, the ROC analysis showed that a change in IVC min $<38 \%$ was the best predictor for rehospitalization both in overall population and in IHD group. Similar results were observed in the IHD subgroup (Table S4).

\section{Discussion}

Our results showed that the early re-admission is quite frequent in patients with CHF and hospitalized for ADHF. Although the re-admission rate in this study was lower than previously reported for patients with $\mathrm{CHF}$, both at 30 days (12\%) [29] as well as at 60 days (16\%) [37], we observed a higher re-admission rate in HF due to IHD as compared to nonIHD. These prognostic differences between different HF aetiologies confirmed previous studies [38, 39] and suggested the urgent need of improving management of HF due to IHD. The identification of biomarkers or surrogate parameters of

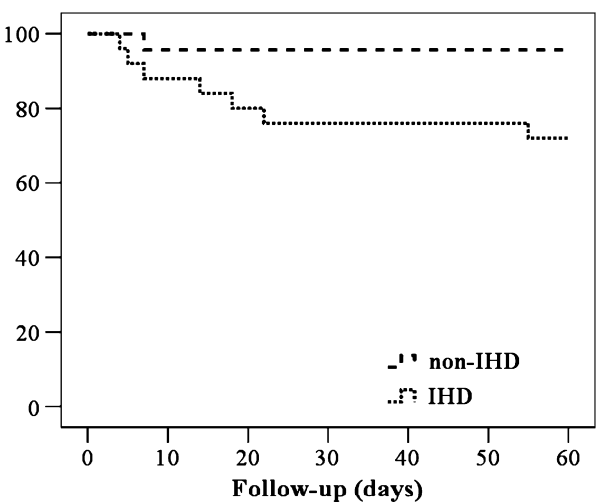

Figure 2 Kaplan-Meier curve analysis for re-admission for ADHF at 60 days after discharge. Trend for re-admission (60 days follow-up) in patients admitted with primary diagnosis of acute decompensated heart failure (ADHF). The curve shows a statistically significative difference (Log Rank $\chi^{2}=4.65$; $P=0.03$ ) between the patients with heart failure secondary to chronic ischaemic heart disease (IHD) or non-IHD. 
Table 2 Adjusted hazard ratio for ADHF 60 days re-admission

\begin{tabular}{|c|c|c|c|c|c|}
\hline 60 days ADHF re-admission adjusted for gender and uric acid at discharge & HR & $\begin{array}{l}95 \% \mathrm{Cl} \\
\text { Lower }\end{array}$ & Upper & Wald $\chi^{2}$ & $P$-value \\
\hline \multicolumn{6}{|l|}{ Overall } \\
\hline $\log (N T-p r o B N P)$ discharge & 2.029 & $1 \cdot 136$ & 3.620 & 5.721 & 0.017 \\
\hline IVC max discharge & $4 \cdot 750$ & $1 \cdot 205$ & $18 \cdot 728$ & 4.954 & 0.026 \\
\hline IVC min discharge & 3.511 & $1 \cdot 277$ & $9 \cdot 654$ & 5.924 & 0.015 \\
\hline IVC collapsibility discharge & 0.967 & 0.936 & 0.998 & $4 \cdot 226$ & 0.040 \\
\hline$\%$ changes IVC max & 0.947 & 0.913 & 0.981 & $8 \cdot 818$ & 0.003 \\
\hline$\%$ changes IVC min & 0.965 & 0.943 & 0.986 & 10.094 & 0.001 \\
\hline \multicolumn{6}{|l|}{ IHD } \\
\hline IVC max discharge & 3.825 & 0.824 & $17 \cdot 748$ & $2 \cdot 934$ & 0.087 \\
\hline IVC min discharge & 4.983 & 1.379 & 18.002 & 6.004 & 0.014 \\
\hline IVC collapsibility discharge & 0.903 & 0.792 & 1.029 & $2 \cdot 333$ & 0.127 \\
\hline$\%$ changes IVC max & 0.918 & 0.853 & 0.988 & $5 \cdot 227$ & 0.022 \\
\hline$\%$ changes IVC min & 0.957 & 0.929 & 0.987 & 8.020 & 0.005 \\
\hline
\end{tabular}

The bold values identify the statistically significant results $(P<0.05)$.

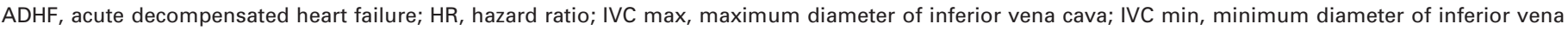
cava; IHD, chronic ischaemic heart disease.

subclinical congestion predicting re-hospitalization in IHD could be a very appropriate approach. At this regard, our study not only confirmed prognostic value of NT-proBNP and HCU IVC diameters and collapsibility [29] in predicting early re-admission for $\mathrm{ADHF}$, but also extended this predictive power up to 60 days. This insight is in partial agreement with what has been described recently by Pellicori and coworkers [40]. However, for our knowledge, this study first recognizes that IVC dimensions, in contrast to NT-proBNP, are the stronger predictors of re-admission in patients with HF due to IHD. Starting from a recent observation that relative change in NT-proBNP level predicts the risk of cardiovascular congestion in haemodialysis patients [41], we also showed that, besides the standard measurements, the change of IVC min from admission to discharge resulted as the best re-admission predictor in overall HF cohort and, in particular, in patients with IHD. Despite several limitations described below, our pilot study suggests that the HCU assessment of IVC at both admission and discharge might be useful to better recognize subclinical volume overload (even better than natriuretic peptide) and predict rehospitalization for ADHF mainly in patients with IHD (a HF subcategory who have more complex management and worse outcomes). This study has several limitations. Firstly, the low number of patients recruited $(n=48)$ and the few events observed $(n=8)$ may affect the

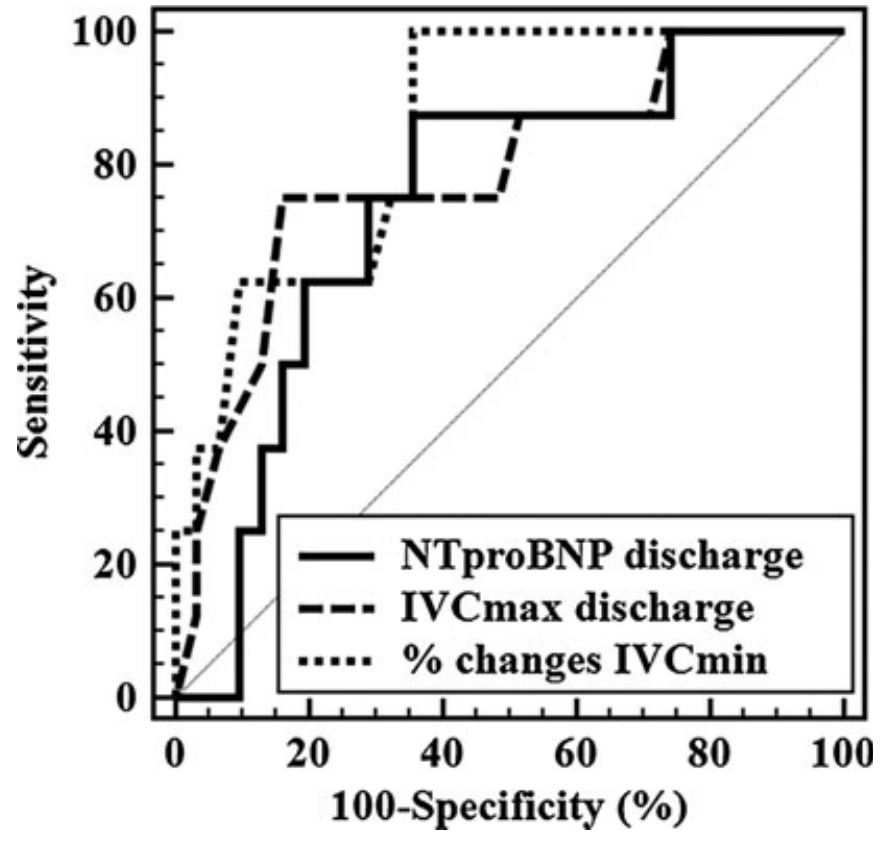

Figure 3 Receiver operator curve (ROC) for ADHF re-admission at 60 days after discharge in patients with IHD. ROC for log(NTproBNP) at discharge, IVC max at discharge and $\%$ change in IVC min from admission are shown. 
clinical relevance of our results so that it may be considered a pilot study. Thus, we need larger clinical trials confirming our results and the previous studies providing same results with similar sample size [29, 42, 43]. Moreover, the cohort of HF patients was recruited in a single hospital, so that the overall heart failure community might be not truly represented. On the other hand, this limitation allowed us to potentially reduce the risk of bias related to different clinical management and operator-dependent ultrasound measurements (only one MD performed all the IVC assessments). Finally, we based the classification of IHD and non-IHD only on anamnestic, EKG and echocardiographic reports. Considering that no additional invasive examinations were performed in this study, we might have misclassified non-IHD or IHD heart failure. However, our rationale of this point was based on previous published studies investigating patients with IHD and non-IHD without performing any invasive examination of coronaries [44-46]. In conclusion, our results support recent evidences suggesting that $\mathrm{CHF}$ has to be considered as a heterogeneous cluster of diseases with different prognosis and responsiveness to treatment. We showed that patients with IHD had an increased re-admission rate for ADHF as compared to non-IHD without any other clinical or laboratory difference at admission or discharge. In this regard, the assessment of IVC over times should be encouraged starting from the admission, considering its usefulness to recognize a cardiogenic dyspnoea [47] and the safety of ultrasound approach. However, the main advantage may be the feasibility of IVC assessment even for noncardiologists. In particular, Mjølstad and coworkers have recently demonstrated that medical residents may perform a correct bedside ultrasound examination of the heart (including IVC) only after 3 months of training [25]. Likewise, David Martin and colleagues showed that hospitalist achieved a diagnostic accuracy of 91\% in IVC assessment only after a mean of 7 weeks training [24]. We believe that these results deserve further study with larger sample size and also randomized trials, to confirm these insights and to evaluate the cost-effectiveness of this ultrasound approach, especially compared with serum natriuretic peptide assay.

\section{Acknowledgements}

The authors Dr. F. Montecucco, Dr N. Vuilleumier and Prof. F. Mach equally contributed as last authors to this work. This study was supported by European Commission (FP7-INNOVATION I HEALTH-F2-2013-602114; Athero-B-Cell: Targeting and exploiting B cell function for treatment in cardiovascular disease). This work was supported by Swiss National Science Foundation Grants to Dr. F. Montecucco (32003B_134963/1), to Dr N. Vuilleumier (310030_140736) and to Prof. F. Mach (310030_118245).

\section{Conflict of interest}

None to be declared.

\section{Address}

Department of Internal Medicine, University of Genoa School of Medicine, IRCCS Azienda Ospedaliera Universitaria San Martino-IST Istituto Nazionale per la Ricerca sul Cancro, 6 vilae Benedetto XV, 16132 Genoa, Italy (F. Carbone, M. Bovio, F. Ferrando, A. Scarrone, G. Murialdo, F. Montecucco); Division of Cardiology, Foundation for Medical Researches, Department of Medical Specialties, University of Geneva, 64 avenue de la Roseraie, 1211 Geneva, Switzerland (F. Carbone, A. Quercioli, F. Mach); Departments of Cardio-Nephrology University of Genoa, School of Medicine. IRCCS Azienda Ospedaliera Universitaria San Martino-IST Istituto Nazionale per la Ricerca sul Cancro, 6 viale Benedetto XV, 16132 Genoa, Italy (G. M. Rosa, F. Viazzi); Division of Laboratory Medicine, Department of Genetics and Laboratory Medicine, Geneva University Hospitals, 4 rue Gabrielle-Perret-Gentil, 1205 Geneva, Switzerland (N. Vuilleumier, F. Montecucco).

Correspondence to: Fabrizio Montecucco, MD, PhD, Division of Laboratory Medicine, Department of Genetics and Laboratory Medicine, Geneva University Hospitals, Avenue de la Roseraie 64, 4 rue Gabrielle-Perret-Gentil, 1205 Geneva, Switzerland. Tel.: +41 223827 238; fax: +41 223827 245; e-mail: Fabrizio.montecucco@unige.ch

Received 18 October 2013; accepted 5 January 2014

\section{References}

1 Gheorghiade M, Filippatos G, De Luca L, Burnett J. Congestion in acute heart failure syndromes: an essential target of evaluation and treatment. Am J Med 2006;119:S3-10.

2 Cleland JG, Swedberg K, Follath F, Komajda M, Cohen-Solal A, Aguilar JC et al. The EuroHeart Failure survey programme - a survey on the quality of care among patients with heart failure in Europe. Part 1: patient characteristics and diagnosis. Eur Heart J 2003;24:442-63.

3 Fonarow GC, Abraham WT, Albert NM, Gattis WA, Gheorghiade M, Greenberg B et al. Organized program to initiate lifesaving treatment in hospitalized patients with heart failure (OPTIMIZE-HF): rationale and design. Am Heart J 2004;148: $43-51$.

4 Adams KF Jr, Fonarow GC, Emerman CL, LeJemtel TH, Costanzo MR, Abraham WT et al. Characteristics and outcomes of patients hospitalized for heart failure in the United States: rationale, design, and preliminary observations from the first 100,000 cases in the Acute Decompensated Heart Failure National Registry (ADHERE). Am Heart J 2005;149:209-16.

5 McMurray JJ, Adamopoulos S, Anker SD, Auricchio A, Bohm M, Dickstein $\mathrm{K}$ et al. ESC guidelines for the diagnosis and treatment of acute and chronic heart failure 2012: the Task Force for the Diagnosis and Treatment of Acute and Chronic Heart Failure 2012 of the 
European Society of Cardiology. Developed in collaboration with the Heart Failure Association (HFA) of the ESC. Eur J Heart Fail 2012;14:803-69.

6 Zaya M, Phan A, Schwarz ER. The dilemma, causes and approaches to avoid recurrent hospital readmissions for patients with chronic heart failure. Heart Fail Rev 2012;17:345-53.

7 Parenica J, Spinar J, Vitovec J, Widimsky P, Linhart A, Fedorco M et al. Long-term survival following acute heart failure: the Acute Heart Failure Database Main registry (AHEAD Main). Eur J Intern Med 2013;24:151-60.

8 Jencks SF, Williams MV, Coleman EA. Rehospitalizations among patients in the Medicare fee-for-service program. $N$ Engl J Med 2009;360:1418-28.

9 Ambrosy AP, Pang PS, Khan S, Konstam MA, Fonarow GC, Traver $\mathrm{B}$ et al. Clinical course and predictive value of congestion during hospitalization in patients admitted for worsening signs and symptoms of heart failure with reduced ejection fraction: findings from the EVEREST trial. Eur Heart J 2013;34:835-43.

10 Gheorghiade M, Pang PS, Ambrosy AP, Lan G, Schmidt P, Filippatos $\mathrm{G}$ et al. A comprehensive, longitudinal description of the in-hospital and post-discharge clinical, laboratory, and neurohormonal course of patients with heart failure who die or are re-hospitalized within 90 days: analysis from the EVEREST trial. Heart Fail Rev 2012;17:485-509.

11 Drazner MH, Brown RN, Kaiser PA, Cabuay B, Lewis NP, Semigran MJ et al. Relationship of right- and left-sided filling pressures in patients with advanced heart failure: a 14-year multi-institutional analysis. J Heart Lung Transplant 2012;31:67-72.

12 Drazner MH, Prasad A, Ayers C, Markham DW, Hastings J, Bhella PS et al. The relationship of right- and left-sided filling pressures in patients with heart failure and a preserved ejection fraction. Circ Heart Fail 2010;3:202-6.

13 Vogelsang TW, Jensen RJ, Monrad AL, Russ K, Olesen UH, Hesse B et al. Independent effects of both right and left ventricular function on plasma brain natriuretic peptide. Eur J Heart Fail 2007;9:892-6.

14 Parsonage WA, Galbraith AJ, Koerbin GL, Potter JM. Value of B-type natriuretic peptide for identifying significantly elevated pulmonary artery wedge pressure in patients treated for established chronic heart failure secondary to ischemic or idiopathic dilated cardiomyopathy. Am J Cardiol 2005;95:883-5.

15 Blair JE, Brennan JM, Goonewardena SN, Shah D, Vasaiwala S, Spencer KT. Usefulness of hand-carried ultrasound to predict elevated left ventricular filling pressure. Am J Cardiol 2009;103:246-7.

16 Goonewardena SN, Blair JE, Manuchehry A, Brennan JM, Keller M, Reeves $\mathrm{R}$ et al. Use of hand carried ultrasound, B-type natriuretic peptide, and clinical assessment in identifying abnormal left ventricular filling pressures in patients referred for right heart catheterization. J Card Fail 2010;16:69-75.

17 Bruce CJ, Montgomery SC, Bailey KR, Tajik J, Seward JB. Utility of hand-carried ultrasound devices used by cardiologists with and without significant echocardiographic experience in the cardiology inpatient and outpatient settings. Am J Cardiol 2002;90:1273-5.

18 DeCara JM, Lang RM, Koch R, Bala R, Penzotti J, Spencer KT. The use of small personal ultrasound devices by internists without formal training in echocardiography. Eur J Echocardiogr 2003;4:141-7.

19 Alexander JH, Peterson ED, Chen AY, Harding TM, Adams DB, Kisslo JA Jr. Feasibility of point-of-care echocardiography by internal medicine house staff. Am Heart J 2004;147:476-81.

20 Dalen H, Haugen BO, Graven T. Feasibility and clinical implementation of hand-held echocardiography. Expert Rev Cardiovasc Ther 2013;11:49-54.
21 Brennan JM, Blair JE, Goonewardena S, Ronan A, Shah D, Vasaiwala $\mathrm{S}$ et al. A comparison by medicine residents of physical examination versus hand-carried ultrasound for estimation of right atrial pressure. Am J Cardiol 2007;99:1614-6.

22 Lucas BP, Candotti C, Margeta B, Evans AT, Mba B, Baru J et al. Diagnostic accuracy of hospitalist-performed hand-carried ultrasound echocardiography after a brief training program. J Hosp Med 2009;4:340-9.

23 Kajimoto K, Madeen K, Nakayama T, Tsudo H, Kuroda T, Abe T. Rapid evaluation by lung-cardiac-inferior vena cava (LCI) integrated ultrasound for differentiating heart failure from pulmonary disease as the cause of acute dyspnea in the emergency setting. Cardiovasc Ultrasound 2012;10:49.

24 David Martin L, Ziegelstein RC, Howell EE, Martire C, Hellmann DB, Hirsch GA. Hospitalists' ability to use hand-carried ultrasound for central venous pressure estimation after a brief training intervention: a pilot study. J Hosp Med 2013;8:711-4.

25 Mjolstad OC, Andersen GN, Dalen H, Graven T, Skjetne K, Kleinau $\mathrm{JO}$ et al. Feasibility and reliability of point-of-care pocket-size echocardiography performed by medical residents. Eur Heart J Cardiovasc Imaging 2013;14:1195-202.

26 International statistical classification od diseases and related health problems (ICD-10). 2010.

27 Felker GM, Shaw LK, O'Connor CM. A standardized definition of ischemic cardiomyopathy for use in clinical research. J Am Coll Cardiol 2002;39:210-8.

28 Muzzarelli S, Leibundgut G, Maeder MT, Rickli H, Handschin R, Gutmann $\mathrm{M}$ et al. Predictors of early readmission or death in elderly patients with heart failure. Am Heart J 2010;160:308-14.

29 Goonewardena SN, Gemignani A, Ronan A, Vasaiwala S, Blair J, Brennan JM et al. Comparison of hand-carried ultrasound assessment of the inferior vena cava and N-terminal pro-brain natriuretic peptide for predicting readmission after hospitalization for acute decompensated heart failure. JACC Cardiovasc Imaging 2008;1:595-601.

30 Gheorghiade M, Vaduganathan M, Fonarow GC, Bonow RO. Rehospitalization for heart failure: problems and perspectives. J Am Coll Cardiol 2013;61:391-403.

31 Gottdiener JS, Bednarz J, Devereux R, Gardin J, Klein A, Manning $\mathrm{WJ}$ et al. American Society of Echocardiography recommendations for use of echocardiography in clinical trials. J Am Soc Echocardiogr 2004;17:1086-119.

32 Schou M, Gustafsson F, Kjaer A, Hildebrandt PR. Long-term clinical variation of NT-proBNP in stable chronic heart failure patients. Eur Heart J 2007;28:177-82.

33 DeLong ER, DeLong DM, Clarke-Pearson DL. Comparing the areas under two or more correlated receiver operating characteristic curves: a nonparametric approach. Biometrics 1988;44:837-45.

34 Papakonstantinou NA, Stamou MI, Baikoussis NG, Goudevenos J, Apostolakis E. Sex differentiation with regard to coronary artery disease. J Cardiol 2013;62:4-11.

35 Kawada T. Serum uric acid and ischemic heart disease incidence. Int J Cardiol 2012;154:381.

36 Hamaguchi S, Furumoto T, Tsuchihashi-Makaya M, Goto K, Goto D, Yokota $\mathrm{T}$ et al. Hyperuricemia predicts adverse outcomes in patients with heart failure. Int J Cardiol 2011;151:143-7.

37 O'Connor CM, Stough WG, Gallup DS, Hasselblad V, Gheorghiade M. Demographics, clinical characteristics, and outcomes of patients hospitalized for decompensated heart failure: observations from the IMPACT-HF registry. I Card Fail 2005;11:200-5.

38 Rossi JS, Flaherty JD, Fonarow GC, Nunez E, Gattis Stough W, Abraham WT et al. Influence of coronary artery disease and 
coronary revascularization status on outcomes in patients with acute heart failure syndromes: a report from OPTIMIZE-HF (Organized Program to Initiate Lifesaving Treatment in Hospitalized Patients with Heart Failure). Eur J Heart Fail 2008;10:1215-23.

39 Vidal-Perez R, Otero-Ravina F, Franco M, Rodriguez Garcia JM, Linares Stolle R, Esteban Alvarez R et al. Determinants of cardiovascular mortality in a cohort of primary care patients with chronic ischemic heart disease. BARBANZA Ischemic Heart Disease (BARIHD) study. Int J Cardiol 2013;167:442-50.

40 Pellicori P, Carubelli V, Zhang J, Castiello T, Sherwi N, Clark AL et al. IVC diameter in patients with chronic heart failure: relationships and prognostic significance. JACC Cardiovasc Imaging 2013;6:16-28

41 Pastural-Thaunat M, Ecochard R, Boumendjel N, Abdullah E, Cardozo C, Lenz A et al. Relative Change in NT-proBNP Level: an Important Risk Predictor of Cardiovascular Congestion in Haemodialysis Patients. Nephron Extra 2012;2:311-8.

42 De Vecchis R, Ariano C, Fusco A, Ciccarelli A, Cioppa C, Giasi A et al. Ultrasound evaluation of the inferior vena cava collapsibility index in congestive heart failure patients treated with intravenous diuretics: new insights about its relationship with renal function: an observational study. Anadolu Kardiyol Derg 2012;12:391-400.

43 Hebl V, Zakharova MY, Canoniero M, Duprez D, Garcia S. Correlation of natriuretic peptides and inferior vena cava size in patients with congestive heart failure. Vasc Health Risk Manag 2012;8:213-8.

44 Zannad F, Briancon S, Juilliere Y, Mertes PM, Villemot JP, Alla F et al. Incidence, clinical and etiologic features, and outcomes of advanced chronic heart failure: the EPICAL Study. Epidemiologie de l'Insuffisance Cardiaque Avancee en Lorraine. J Am Coll Cardiol 1999;33:734-42.

$45 \mathrm{Ng} \mathrm{AC}$, Sindone AP, Wong HS, Freedman SB. Differences in management and outcome of ischemic and non-ischemic cardiomyopathy. Int J Cardiol 2008;129:198-204.

46 Teng TH, Hung J, Knuiman M, Stewart S, Arnolda L, Jacobs I et al. Trends in long-term cardiovascular mortality and morbidity in men and women with heart failure of ischemic versus non-ischemic aetiology in Western Australia between 1990 and 2005. Int J Cardiol 2012;158:405-10.

47 Anderson KL, Jenq KY, Fields JM, Panebianco NL, Dean AJ. Diagnosing heart failure among acutely dyspneic patients with cardiac, inferior vena cava, and lung ultrasonography. Am J Emerg Med 2013;31:1208-14.

\section{Supporting Information}

Additional Supporting Information may be found in the online version of this article:

Table S1. Clinical characteristics of study populations at admission.

Table S2. Echocardiographic characteristics of study patients.

Table S3. Crude hazard ratio for ADHF 60 days re-admission. Table S4. ROC analysis of re-admission for ADHF at 60 days from discharge. 